\title{
Unprecedented interweaving of single-helical and unequal double-helical chains into chiral metal-organic open frameworks with multiwalled tubular structures $\dagger$
}

\author{
Su-Na Wang, ${ }^{a}$ Hang Xing, ${ }^{a}$ Yi-Zhi Li, ${ }^{a}$ Junfeng Bai, ${ }^{* a}$ Manfred Scheer, ${ }^{* b}$ Yi Pan*a and Xiao-Zeng You ${ }^{a}$ \\ Received (in Cambridge, UK) 25th January 2007, Accepted 15th February 2007 \\ First published as an Advance Article on the web 7th March 2007 \\ DOI: $10.1039 / b 701186 e$
}

Two novel chiral metal-organic open frameworks with unprecedented multiwalled tubular channels and interweaving of single-helical and unequal double-helical units were reported, which possess novel four-connected $4^{2} 8^{3} 10$ topology and represent the first examples of functional metal-organic frameworks based on both flexible tripodal and flexible bisdentate ligands.

Rapid development of metal-organic frameworks has been made in recent years not only for their potential applications but also for fascinating architectures and topologies. ${ }^{1,2}$ Of higher interest is the exploration of highly symmetrical multitopic ligands, which may adjust various coordination modes to satisfy the requirements of assembly process and lead to interesting structural motifs, especially those with high symmetries and extreme beauty. ${ }^{3}$ Meanwhile, helicity, as an active field of crystal engineering, has also been successfully introduced into metallo-supramolecular architectures. ${ }^{4}$ Discrete helices or low-dimensional helical coordination polymers have been extensively reported, ${ }^{5}$ while control of assembly of the helical units into a multi-helical-array is still a great challenge. Only few examples have been reported concerning harmonic single and multiple helices in one coordination complex, all of which are involved with equal helical chains. ${ }^{6}$ To the best of our knowledge, no examples in which unequal coaxial helical chains intertwine with each other were found to exist in a stable crystalline solid, particularly those with the same compositions.

Moreover, tubular architectures have mushroomed in the fields of materials science, nanotechnology, molecular sieves, ion sensors, and fluidic transport systems. Much effort has been made in inorganic and organic tubular structures, ${ }^{7}$ but the corresponding architectures of coordination frameworks are still in their infancy. Though coordination networks with large and single-walled tubes are documented, ${ }^{8}$ those with multiwalled tubes remain unexplored up to now.

Along with our work of assembly of supramolecular polymers based upon highly symmetrical ligands, ${ }^{9}$ herein we report two unprecedented three-dimensional (3-D), four-connected $4^{2} 8^{3} 10$

${ }^{a}$ State Key Laboratory of Coordination Chemistry, School of Chemistry and Chemical Engineering, Nanjing University, Nanjing, 210093, P. R. China.E-mail: bjunfeng@nju.edu.cn; yipan@nju.edu.cn;

Fax: (+86) 25-8331-4502

${ }^{b}$ Institut für Anorganische Chemie der Universität Regensburg, 93040, Regensburg, Germany.

E-mail: manfred.scheer@chemie.uni-egensburg.de

$\dagger$ Electronic supplementary information (ESI) available: Asymmetric unit, other supplementary figures, TGA, XRPD of $\mathbf{1}$ and 2. See DOI: 10.1039/ b701186e architectures $\left[\mathrm{Ni}_{2}(\mathrm{HTTG})_{2}(\mathrm{bpp})_{2}\left(\mathrm{H}_{2} \mathrm{O}\right)_{9}\right](\mathbf{1})$ and $\left[\mathrm{Co}_{2}(\mathrm{HTTG})_{2^{-}}\right.$ (bpp) $\left.)_{2}\left(\mathrm{H}_{2} \mathrm{O}\right)_{9}\right]$ (2), with multiwalled tubular channels and interweaving of single-helical and novel unequal double-helical chains from a versatile flexible tripodal ligand $\mathrm{H}_{3}$ TTG $\left(N, N^{\prime}, N^{\prime \prime}-1,3,5-\right.$ triazine-2,4,6-triyltrisglycine) and an exo-bisdentate ligand bpp (1,3-bis(4-pyridyl)propane) \& X-Ray crystallographic analysis $\S$ indicates the two complexes are isomorphous and therefore only the structure of complex $\mathbf{1}$ is discussed in detail. It crystallized in the chiral space group $P 6_{5} 22$ and the fundamental asymmetric unit consists of two crystallographically independent $\mathrm{Ni}^{2+}$ ions (Fig. S1, ESI $\dagger$ ). Both metal centers lie on twofold axes and possess an octahedral geometry with the same coordination environment of $\mathrm{Ni}\left\{\mathrm{N}_{2} \mathrm{O}_{4}\right\}$ moieties, showing coordination isomerism with different arrangement of the ligated atoms.

The most striking feature of complex 1 is interweaving of singlehelical and novel unequal double-helical chains formed by complicated linkages of the tripodal ligands and bpp ligands between different Ni(II) centers (Fig. 1, Fig. S2, ESI $\dagger$ ). In order to interpret the whole network clearly, separate description of several
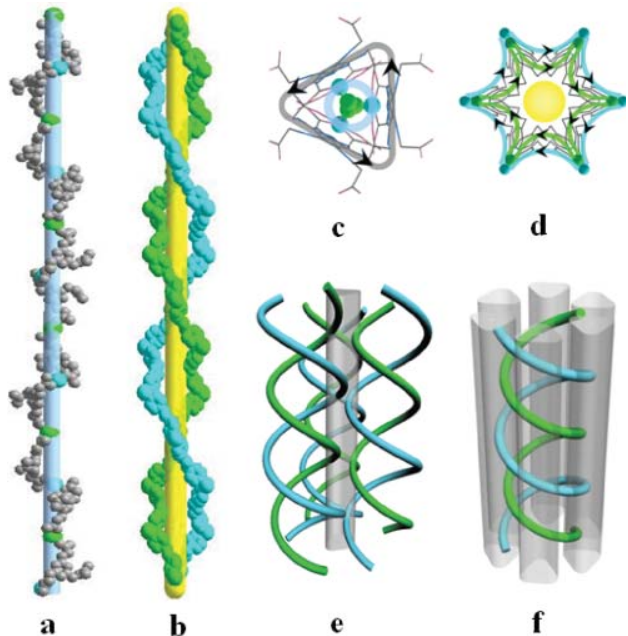

Fig. 1 (a) View of $\mathbf{A}$ along the $b$ axis. Ni1, sky blue; Ni2, green; C, O, N, gray. (b) View of $\mathbf{B}$ and $\mathbf{C}$ along the $b$ axis. Two different chains are marked as sky blue (-Nil-bpp-) (B) and green (-Ni2-bpp-) (C), respectively. (c) View of $\mathbf{A}$ along the $c$ axis. Black arrows: the orientation of the right-handed helix. (d) View of $\mathbf{B}$ and $\mathbf{C}$ along the $c$ axis. Arrows: orientations of the left-handed helical chains. (e) Simple representation of the linkage of one A with three pairs of B and C. A, Gray trigonal prism; $\mathbf{B}$, sky blue chain; $\mathbf{C}$, green chain. (f) The linkage of one pair of $\mathbf{B}$ and $\mathbf{C}$ with six A. 
relative chains is needed, i.e., metal-acid (A) and metal-base (B and $\mathbf{C}$ ) chains.

The single helix $\mathbf{A}$ is made up of metal centers and incompletely deprotonated ligands HTTG. Two carboxylate groups of the ligands are bonded to $\mathrm{Ni1}$ and $\mathrm{Ni} 2$ centers in monodentate fashion, giving rise to an infinite right-handed helical chain (Ni1HTTG-Ni2-HTTG) $)_{n}$ along the $c$ axis, which is generated along the crystallographic $3_{1}$ screw axis with a long pitch of 62.471(3) $\AA$ based on the repeated unit containing six metal centers and six ligands (Fig. 1(a) and (c), Fig. S3, ESI + ). Notably, the flexible tripodal multicarboxylate ligand acts as a bisdentate linker in constructing the helical chains. The double-stranded helices $\mathbf{B}$ and C consist of two intertwined chains of bpp ligands bounded by metal centers. Bpp is also situated on twofold axes and two types of these ligands adopt trans-trans (TT) conformations with different angles of the pyridine rings to bridge Ni1 and $\mathrm{Ni} 2$ centers along the $c$ axis, respectively. As a result, two unequal coaxial left-handed helical chains possessing $6_{5}$ screw axis are formed (Fig. 1(b) and (d), Fig. S4, ESI $\dagger$ ). They possess the same long pitches as that of $\mathbf{A}$ and twist with each other to form doublestranded helical structures. Viewed down the $c$ axis, they are arranged as two beautiful homocentric hexagrams. Apparently, this is exceptional for general multiple helical structures, which are usually intertwisted by the same helical chains.

Interweaving of single-helical with double-helical chains leads to an unprecedented three-dimensional (3D) network. Each A with crystallographic $3_{1}$ screw axis assemblies three pairs of double helices in $\mathbf{B}_{1} \mathbf{C}_{3} \mathbf{B}_{2} \mathbf{C}_{1} \mathbf{B}_{3} \mathbf{C}_{2}$ sequence $\left(\mathbf{B}_{n} \mathbf{C}_{n}\right.$ represents one pair of double-stranded helical chains) through alternating Ni1 and $\mathrm{Ni} 2$ centers (Fig. 1e, Fig. S5a and S6). In contrast, each B with crystallographic $6_{5}$ screw axis coils through six neighboring metalacid chains during one helical period, and so does $\mathbf{C}$ (Fig. 1(f) and Fig. S5b, ESI $\dagger$ ). Remarkably, compared with rare examples about the interweaving of different helical ribbons, this unusual helical motif in this complex may be one of the most complicated entanglement structures ever found in coordination chemistry of helical arrangements. ${ }^{6 b, d}$ Moreover, if each metal center can be considered as a node and the two kinds of ligands as linear spacers, the whole structure is a novel four-connected chiral network with a Schäfli symbol of $4^{2} 8^{3} 10$ with long vertex symbol $4.4 .8^{2} \cdot 8^{2} \cdot 8^{8} \cdot 10^{12}$ (Fig. 2). ${ }^{10}$

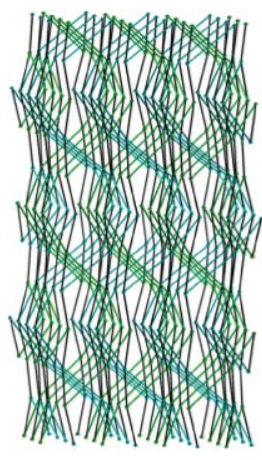

(a)

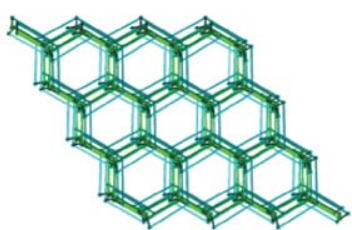

(b)

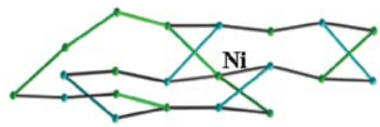

(c)
Fig. 2 (a) An unusual topology of the four-connected network of complex 1 viewed along the $b$ axis. (b) The topology along the $c$ axis. (c) A convenient view of the essential circuits of the vertex.
Another remarkable character of the complex is formation of extraordinary one-dimensional multiwalled tubular channels along the crystallographic $c$ axis (Fig. 3 and Fig. S7, ESI $\dagger$ ). A cross section shows that the channel is composed of three parts: trunk, inner shell and outer shell. Firstly, the open-ended channel is supported by six A in hexagonal arrangement, just like the trunk. Secondly, the inner shell is generated by one pair of $\mathbf{B}$ and $\mathbf{C}$ twisting together with a minimum internal diameter of $c a .5 \AA$ without considering the van der Waals contacts. Thirdly, another two metal-base ribbons are complementary with $\mathbf{B}$ and $\mathbf{C}$, forming the outer shell. Interestingly, they are not built up of separate helical chains, but of segments from six pairs of double helical chains of adjacent channels with average diameter of $c a .16 \AA$ between the utmost atoms across the section. As a consequence, one multiwalled tubular channel is obtained and each channel shares the outer shells with the other six to build three-dimensional frameworks. In the known nanotubular coordination polymers, the channels always have mono-layered walls, ${ }^{8}$ and the complex we report represents the first crystalline multiwalled tubular structure.

Additionally, rare helical water chains fill in the chiral channels (Fig. S8 and S9, ESI $\dagger$ ) and the total solvent-accessible volume of the channels in the unit cell is $1179.6 \AA^{3}$, which accounts for $13.8 \%$ of the total cell volume as calculated by PLATON. ${ }^{11}$

The formation of these special structures in $\mathbf{1}$ and $\mathbf{2}$ may be due to the adaptable combination of the metal centers and two relatively flexible ligands. $\mathrm{H}_{3}$ TTG is a novel flexible tripodal multicarboxylate ligand containing additional $\mathrm{NHCH}_{2}$ groups and may adopt many acidity-dependent conformations, both symmetrical and asymmetrical. Meanwhile, the highly flexible bpp ligand can exhibit different conformations with respect to the relative orientations of the $\mathrm{CH}_{2}$ groups. Both ligands could cooperate with each other by adjusting their conformations to generate coordination isomerism of the metal centers. The undeprotonated carboxylate groups of the tripodal ligands are also involved in hydrogen bonding interactions, which may also play important roles in constructing the final frameworks.

To further identify the stability of the whole frameworks, thermal gravimetric analysis (TGA) and X-ray diffraction

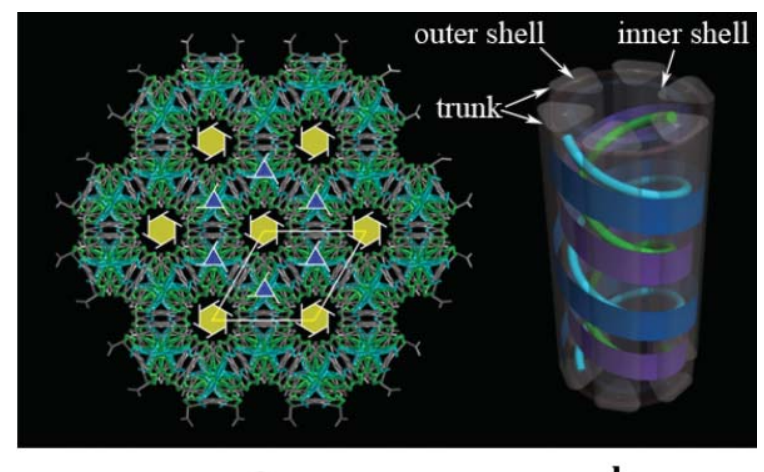

a

b

Fig. 3 (a) View of the whole network along the $c$ axis, the yellow hexagons and blue triangular ones represent the crystallographic $6_{5}$ and $3_{1}$ screw axes, respectively. (b) View of the multiwalled channel. Trunk: six gray triangular prisms; Inner shell: blue cylinder; Outer shell: brick red cylinder. The light blue and green double-helical chains represent $\mathbf{B}$ and $\mathbf{C}$. The blue and purple bands represent ribbons of the metal-base chains constructing the outer shell. 
(XPRD) measurements were performed (Fig. S10 and S11, ESI $\dagger$ ). TGA curves for both complexes revealed the release of the solvent and the coordinated water molecules occurs in the range 25-140 ${ }^{\circ} \mathrm{C}$, and the decomposition of the whole networks begins above $200{ }^{\circ} \mathrm{C}$. XPRD measurements suggested the desolvated solids of these complexes may be potential reversible adsorbent materials for water molecules. In addition, nonlinear optical (NLO) properties of the complexes have also been investigated. Second harmonic generation experimental results show that both complexes display modest SHG efficiencies approximately equivalent to that of potassium dihydrogen phosphate (KDP). As far as we know, most SHG-active materials are condensed structures ${ }^{12}$ and microporous metal-organic frameworks with such properties are uncommon. In addition, in principle any MOF can crystallize in a chiral space group as a result of a particular spatial disposition of all the building units, while the bulk samples of the MOFs built from achiral components tend to contain both enantiomorphs and are thus racemic even though the single crystals are chiral, ${ }^{13}$ which is consistent with our CD experiments on bulk samples.

In summary, two novel metal-organic open frameworks with unprecedented multiwalled tubular channels and interweaving of single-helical and unequal double-helical units were reported. They display special chiral four-connected $4^{2} 8^{3} 10$ networks and exhibit interesting reversible crystal-to-amorphous transformation properties and modest SHG efficiencies. These compounds represent the first examples of functional metal-organic open frameworks based on both flexible tripodal and flexible bisdentate ligands, demonstrating that combination of cooperative flexible ligands can lead to fascinating architectures with interesting properties and may further contribute to understanding of assembly and recognition process in coordination chemistry.

We gratefully acknowledge the financial support of the Talent Foundation of Jiangsu Province (BK2006513), the Major State Basic Research Development Program (Grant No. 2006CB806104), Twenty-one Century Talent Foundation of the Ministry of Education, Foundation for the Returnee of the Ministry of Education, and National Natural Science Foundation of China (No. 20301010).

\section{Notes and references}

\$ Synthesis of complexes 1 and 2: A mixture of metal salts $(0.1 \mathrm{mmol}$; $\mathrm{Ni}(\mathrm{OAc})_{2} \cdot 6 \mathrm{H}_{2} \mathrm{O}, 0.028 \mathrm{~g}$; or $\left.\mathrm{CoCl}_{2} \cdot 6 \mathrm{H}_{2} \mathrm{O}, 0.024 \mathrm{~g}\right), \mathrm{H}_{3} \mathrm{TTG}(0.1 \mathrm{mmol}$, $0.030 \mathrm{~g})$, bpp $(0.3 \mathrm{mmol}, 0.054 \mathrm{~g})$ and $\mathrm{H}_{2} \mathrm{O}(10 \mathrm{~mL})$ was sealed in a $25-\mathrm{mL}$ stainless steel reactor with a Teflon liner and heated at $120{ }^{\circ} \mathrm{C}$ for 2 days, and then cooled to room temperature.

\$ Crystal data for the two complexes: 1: $\mathrm{C}_{44} \mathrm{H}_{66} \mathrm{~N}_{16} \mathrm{O}_{21} \mathrm{Ni}_{2}, 1272.55$, hexagonal, $P 6_{5} 22, a=b=12.5611(4), c=62.471(3) \AA, V=8536.2(6) \AA^{3}$, $Z>=6, F(000)=3996, D_{\mathrm{c}}=1.485 \mathrm{Mg} \mathrm{m}^{-3}, w R_{2}=0.1409, S=1.033, R_{1}=$ 0.0543, Flack $=0.17 .2: \mathrm{C}_{44} \mathrm{H}_{66} \mathrm{~N}_{16} \mathrm{O}_{21} \mathrm{Co}_{2}, 1272.99$, hexagonal, $P 6_{5} 22, a=$ $b=12.5764(6), c=62.941(4) \AA, V=8621.4(8) \AA^{3}, Z=6, F(000)=3984$, $D_{\mathrm{c}}=1.471 \mathrm{Mg} \mathrm{m}^{-3}, w R_{2}=0.1179, S=1.019, R_{1}=0.0468$, Flack $=0.04$. CCDC 633773-633774. For crystallographic data in CIF or other electronic format see DOI: 10.1039/b701186e
1 (a) J. M. Lehn, Supramolecular Chemistry: Concepts and Perspectives, VCH, Weinheim, 1995; (b) Comprehensive Supramolecular Chemistry, ed. J. M. Lehn, J. L. Atwood, E. D. J. Davis, D. D. MacNicol and F. Vögtle, Pergamon, Oxford, 1990-1996. vol. 1-11 .

2 (a) O. M. Yaghi, H. Li, C. Davis, D. Richardson and T. L. Groy, Acc. Chem. Res., 1998, 31, 474 484; (b) C. Janiak, Dalton Trans., 2003, 2781-2804; (c) G. S. Papaefstathiou and L. R. MacGillivray, Coord. Chem. Rev., 2003, 246, 169-184; (d) D. Bradshaw, J. B. Claridge, E. J. Cussen, T. J. Prior and M. J. Rosseinsky, Acc. Chem. Res., 2005, 38, 273-282; (e) S. Kitagawa, S. Noro and T. Nakamura, Chem. Commun., 2006, 7, 701-707.

3 (a) A. Markus, Angew. Chem., Int. Ed., 1999, 38, 3463-3465; (b) S. R. Seidel and P. J. Stang, Acc. Chem. Res., 2002, 35, 972-983; (c) D. N. Dybtsev, H. Chun and K. Kim, Chem. Commun., 2004, 1594-1595; (d) M. Yoshizawa, T. Kusukawa, M. Kawano, T. Ohara, I. Tanaka, K. Kurihara, N. Niimura and M. Fujita, J. Am. Chem. Soc., 2005, 127, 2798-2799.

4 Review articles: $(a)$ C. Piguet, G. Bernardinelli and G. Hopfgartner, Chem. Rev., 1997, 97, 2005-2062; (b) G. F. Swiegers and T. J. Malefetse, Chem. Rev., 2000, 100, 3483-3537; (c) M. Albrecht, Chem. Rev., 2001, 101, 3457-3497; (d) B. Kesanli and W. B. Lin, Coord. Chem. Rev., 2003, 246, 305-326; (e) L. Han and M. C. Hong, Inorg. Chem. Commun., 2005, 8, 406-419; (f) M. Albrecht, Angew. Chem., Int. Ed., 2005, 44, $6448-6451$.

5 (a) V. Berl, I. Huc, R. G. Khoeuy, M. J. Krische and J. M. Lehn, Nature, 2000, 407, 720-723; (b) X. M. Chen and G. F. Liu, Chem. Eur: J., 2002, 8, 4811-4817; (c) L. Pan, K. M. Adams, H. E. Hernandez, X. Wang, C. Zheng, Y. Hattori and K. Kaneko, J. Am. Chem. Soc., 2003, 125, 3062-3067; (d) X. J. Luan, Y. Y. Wang, D. S. Li, P. Liu, H. M. Hu, Q. Z. Shi and S. M. Peng, Angew. Chem., Int. Ed., 2005, 44, 3864-3867; (e) D. R. Xiao, E. B. Wang, H. Y. An, Y. G. Li, Z. M. Su and C. Y. Sun, Chem. Eur. J., 2006, 12, 6528-6541.

6 (a) Y. Cui, S. J. Lee and W. B. Lin, J. Am. Chem. Soc., 2003, 125, 6014-6015; (b) Y. Q. Sun, J. Zhang, Y. M. Chen and G. Y. Yang, Angew. Chem., Int. Ed., 2005, 44, 5814-5817; (c) G. O. Lloyd, J. L. Atwood and L. J. Barbour, Chem. Commun., 2005, 1845-1847; (d) C. Qin, X. L. Wang, E. B. Wang and L. Xu, Inorg. Chem. Commun., 2005, 669-672; (e) S. Q. Zang, Y. Su, C. Y. Duan, Y. Z. Li, H. Z. Zhu and Q. J. Meng, Chem. Commun., 2006, 4997-4999.

7 (a) S. Iijima, Nature, 1991, 354, 56-58; (b) H. W. Zhu, C. L. Xu, D. H. Wu, B. Q. Wei, R. Vajtai and P. M. Ajayan, Science, 2002, 296, 884-886; (c) A. L. Sisson, V. del A. Sanchez, G. Magro, A. M. E Griffin, S. Shah, J. P. H. Charmant and A. P. Davis, Angew. Chem., Int. Ed., 2005, 44, 6878-6881; (d) D. M. Guldi, A. Rahman, V. Sgobba and C. Ehli, Chem. Soc. Rev., 2006, 35, 471-487.

8 (a) M. C. Hong, Y. J. Zhao, W. P. Su, R. Cao, M. Fujita, Z. Y. Zhou and A. S. C. Chan, Angew. Chem., Int. Ed., 2000, 39, 2468-2469; (b) B. Zhao, P. Cheng, Y. Dai, C. Cheng, D. Z. Liao, S. P. Yan, Z. H. Jiang and G. L. Wang, Angew. Chem., Int. Ed., 2003, 42, 934-936; (c) C. Y. Su, A. M. Goforth, M. D. Smith, P. J. Pellechia and H.-C. zur Loye, J. Am. Chem. Soc., 2004, 126, 3576-3586; (d) W. Schmitt, J. P. Hill, S. Malik, C. A. Volkert, I. Ichinose, C. E. Anson and A. K. Powell, Angew. Chem., Int. Ed., 2005, 44, 7048-7053.

9 (a) J. Bai, A. V. Virovets and M. Scheer, Angew. Chem., Int. Ed., 2002, 41, 1737-1740; (b) J. Bai, A. V. Virovets and M. Scheer, Science, 2003, 300, 781-783; (c) B. P. Johnson, F. Dielmann, G. Balázs, M. Sierka and M. Scheer, Angew. Chem., Int. Ed., 2006, 45, 2473-2475; (d) S.-N. Wang, H. Xing, Y.-Z. Li, J. Bai, Y. Pan, M. Scheer and X. Z. You, Eur. J. Inorg. Chem., 2006, 15, 3041-3053.

10 O. V. Dolomanov, A. J. Blake, N. R. Champness and M. Schröder, J. Appl. Crystallogr., 2003, 36, 1283-1284.

11 A. L. Spek, Acta Crystallogr., Sect. A, 1990, 46, C34.

12 (a) S. D. Bella, Coord. Chem. Rev., 2001, 30, 355-366; (b) O. R. Evans and W. B. Lin, Acc. Chem. Res., 2002, 35, 511-522.

13 M. J. Zaworotko and B. Moulton, Chem. Rev., 2001, 101, 1629-1658. 